\title{
Are PDFs still consistent with Tevatron data?
}

\author{
Zack Sullivan ${ }^{1, \star}$ \\ ${ }^{1}$ Department of Physics, Illinois Institute of Technology, Chicago, Illinois 60616-3793, USA
}

\begin{abstract}
As active data taking has moved to the LHC at CERN, more and more LHC data have been included into fits of parton distribution functions. An anomaly has arisen where formerly excellent agreement between theoretical predictions and experiment in single-top-quark production at the Tevatron is no longer quite as good. Is this indicative of a deeper issue?
\end{abstract}

\section{Introduction}

In the years leading up to its discovery at the Fermilab Tevatron $[1,2] t$-channel single-top-quark production played a pivotal role in the development and understanding of improved perturbation theory and heavy quark parton distribution functions (PDFs). The analytic connection between this process and deeply inelastic scattering (DIS) couples single-top to choices made in the extraction of PDFs that imply significant constraints on higher order corrections and scale choices. Furthermore, large logarithms that appear in intermediate steps of next-to-leading order (NLO) and next-to-nextto-leading order (NNLO) calculations undergo delicate cancellations that magnify any errors in the PDFs. Hence, $t$-channel single-top-quark production is one of the most constraining processes on the consistency PDF fits at different orders, and it directly tests the analytic framework of improved perturbation theory.

This paper introduces how modern PDF sets are failing some of the stringent analytic tests set by $t$-channel single-top-quark production, and speculates on some possible reasons for the failures. In Sec. 2 I explain the constraints imposed by this process and why it is such a stringent test. In Sec. 3 I demonstrate how past PDF sets were successful (or failed in calculable ways), but current PDFs from three collaborations CTEQ, NNPDF, and HERAPDF fail by upwards of $5 \sigma$. In Sec. 4 I explore possible explanations, and suggest that we may be seeing first hints that our framework needs to be improved. I conclude with recommendations for where to proceed to solve these issues.

\section{$2 t$-channel single top, scales, and large logarithms}

Data from the CERN Large Hadron Collider (LHC) has become precise enough to require significantly improved theoretical calculations. While the state-of-the-art calculations have shifted to fully differential NNLO, few checks have been performed to confirm that the framework and all of its pieces are both self-consistent and consistent with all previous data. The NNLO calculation of

\footnotetext{
^e-mail: Zack.Sullivan@IIT.edu
} 
$t$-channel single-top-quark production with a stable top quark [3] (see Fig. 1 for a leading-order figure) was recently updated to include NNLO decays of the top quark [4]. When coupled to NNLO PDFs, this enables a comparison to experimental data at the Tevatron and LHC with small theoretical uncertainties.

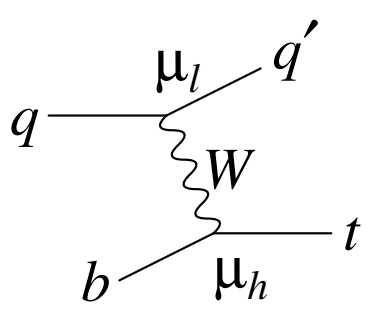

Figure 1. Leading order Feynman diagram for $t$-channel single-top-quark production. The light-quark and heavy-quark sides of the diagram factorize with independent scale $\mu_{l}$ and $\mu_{h}$, respectively.

There is only one problem with the calculation in Refs. [3, 4]: both were performed at fixed factorization $\mu_{F}$ and renormalization $\mu_{R}$ scales set to the top quark mass $m_{t}$. Why is this a problem? Isn't it true that theoretical calculations should be independent of scale choice (or at least push the effect to higher orders)? Not in this case.

$t$-channel single-top-quark production is a special process in that it is analytically identical to double deeply-inelastic-scattering (DDIS) through NLO $[5,6]$. The reason is simply that you cannot exchange a single gluon between the light-quark line $\left(q-q^{\prime}\right.$ in Fig. 1$)$ and the heavy-quark line ( $b-t$ in Fig. 1) and conserve color. In principle you could exchange two gluons at NNLO, but Refs. [3, 5, 7] argue that this is numerically a tiny effect, and so preserves an effective separation between sides of the diagram. Hence, you have DDIS with two sets of independent scales: $\mu_{l}$ and $\mu_{h}$ for the light- and heavy-quark lines, respectively.

This critical feature of this factorization is that when you calculate DIS you must choose the same scale to evaluate your calculation as was chosen to extract the PDFs from DIS data. Otherwise, you do not get back to the data. This means there is a unique choice for $t$-channel single-top: $\mu_{l}=Q^{2}$ and $\mu_{h}=Q^{2}+m_{t}^{2}$, where $Q^{2}$ is the virtuality of the $W$ boson, and the heavy-quark line sees the heavyquark version of DIS [5]. A further observation is since DIS data is used to fit the PDF, the inclusive cross sections calculated at LO, NLO, or NNLO must all be identical — again you are just undoing the fit at each order. This is a very powerful constraint on the PDF fits.

A second important aspect of $t$-channel single-top-quark production is related to its name. If one tries to work in a 4-flavor scheme where there is no $b$ quark in the initial state, a large logarithmic divergence of the form

$$
\alpha_{s} \ln \left(\frac{Q^{2}+m_{t}^{2}}{m_{b}^{2}}\right)
$$

appears at every order in the perturbative series [5]. Numerically this term is close to 1 . Hence, you resum these large logarithms into a $b$ PDF via the DGLAP equation [5]

$$
b\left(x, \mu^{2}\right)=\frac{\alpha_{s}\left(\mu^{2}\right)}{2 \pi} \ln \left(\frac{\mu^{2}}{m_{b}^{2}}\right) \int_{x}^{1} \frac{d z}{z} P_{b g}(z) g\left(\frac{x}{z}, \mu^{2}\right) .
$$

This creates an improved perturbation series in which $b$ degrees of freedom are an intrinsic part of proton structure. In NLO and NNLO calculations, careful subtraction of the resummed logarithms 
Table 1. Inclusive LO and NLO $t$-channel single-top-quark cross section $(t+\bar{t})$ calculated for run II of the Tevatron $(\sqrt{S}=1.96 \mathrm{TeV})$ with various PDFs.

\begin{tabular}{llll} 
PDF & LO $(\mathrm{pb})$ & NLO $(\mathrm{pb})$ & Notes \\
\hline CTEQ 4L/4M & 2.26 & 2.41 & $6 \%$ deviation, known $\alpha_{s}$ bug \\
CTEQ 5L/5M1 & 2.08 & 2.07 & $<0.5 \%$ (bug fixed) \\
CTEQ 6L1/6M & 2.07 & 2.086 & $<0.5 \%$ \\
CTEQ 6L1/6M & 1.83 & 2.086 & Scales set to $m_{t}, 12 \%$ off as expected \\
\hline CTEQ 14 llo/nlo & 2.39 & 2.00 & 20\% LO-NLO deviation! \\
HERAPDF 1.5 lo/nlo & 1.965 & 1.798 & $9.3 \%$ deviation! \\
HERAPDF 2.0 lo/nlo & 1.910 & 1.762 & $8.4 \%$ deviation, NLO 12\% too small \\
NNPDF 3.0 lo/nlo & 2.33 & 2.21 & $5.4 \%$ deviation, NLO 10\% too big \\
\hline
\end{tabular}

leads to rapidly convergent series. However, any small mistake in either the calculation or PDF input (e.g., through faulty DGLAP evolution or poor fits) reintroduces these large logarithms, and formerly delicate cancellations that occur to enforce the equality of the inclusive cross sections between orders lead to large measurable deviations.

$t$-channel single-top-quark production is one of the most sensitive processes for testing the idea of heavy quark PDFs, the resummation framework for improved perturbation theory, and our mapping of the degrees of freedom of the proton onto universal parton distribution functions.

\section{The failure of modern LO and NLO PDFs}

Before updating the NNLO calculation to utilize the DDIS scales, I first updated the calculation of the inclusive $t$-channel cross section with modern PDFs using the public code ZTOP [6]. The ZTOP code was recently interfaced to the new LHAPDF [9] standard for accessing PDFs, and is the standard for LO and NLO $t$-channel production. For this study I focus on results for the Tevatron run II at $1.96 \mathrm{TeV}$, where a combined $\mathrm{CDF}$ and $\mathrm{D} \emptyset$ analysis exists [10].

In the top half of Tab. 1 I show the results for inclusive single top production at the Tevatron calculated with older CTEQ PDFs [11-13] for which there are no NNLO fits. In order to be able to compare with Ref. [10], I use a top quark mass $m_{t}=172.5 \mathrm{GeV}$ and the DDIS scales $\left(\mu_{l}=Q^{2}\right.$, $\left.\mu_{h}=Q^{2}+m_{t}^{2}\right)$. LO means a LO matrix element, with LO PDFs, and $\alpha_{s}\left(M_{Z}\right)=0.130$. NLO means a NLO matrix element, with NLO PDFs, and $\alpha_{s}\left(M_{Z}\right)=0.118$.

The CTEQ 4 PDF sets had a 6\% disagreement between LO and NLO, however a bug in the running of $\alpha_{s}$ was discovered that accounted for the effect. The bug was fixed in CTEQ 5 with little change to the data in the PDF fits, and the LO and NLO agree within the Monte Carlo numerical integration. Everything continued to agree with CTEQ 6, and other distributions not shown, and the CTEQ 6 central value is identical to the combined Tevatron fit [10]. One observation from Tab. 1 is that choosing the top quark mass as a scale (as is done in Refs. [3, 4] and MCFM [14]) induces a large predictable $12 \%$ shift between orders. While the scale dependence formally decreases at higher orders, as it should, there is a shift with respect to the PDF extraction by a term proportional to $\ln \left(Q^{2}+m_{t}^{2} / m_{t}^{2}\right)$. The DDIS scales are necessary here.

In the bottom half of Tab. 1 I show LO and NLO results for the inclusive cross section using modern PDFs, for which NNLO fits exist. Nothing agrees between LO and NLO, and the NLO calculations between PDF sets are inconsistent. Beginning with the latter point, the expected $90 \%$ confidence level NLO PDF uncertainty for this process is $+8.8-7.3 \%$ as calculated with CTEQ 14 [15] PDFs - this is consistent with error calculations using CTEQ 6, HERAPDF, and NNPDF 3.0. 
However, NNPDF [16] and HERAPDF [17] disagree with each other at NLO by $5 \sigma$, with CTEQ 14 splitting the difference! The Tevatron data [10] is not precise enough to rule out NNPDF and HERAPDF, but there is significant tension with the single top data.

While the NLO discrepancies are serious, the formal question addressed here - the consistency between LO and NLO - is a disaster. The CTEQ 14 PDFs, which are closest to the data at NLO, have a $20 \%$ deviation from LO when the difference should be zero. NNPDF 3.0 is shown as having a 5.4\% deviation, but this is an artifact of choosing $m_{t}=172.5 \mathrm{GeV}$. If $m_{t}=175 \mathrm{GeV}$ the discrepancy also grows to double-digits. Even HERAPDF 1.5 and 2 are off by almost 10\%. No modern PDF gets back to the data. One might be tempted to ask these same questions at the LHC where there is more data. Unfortunately, all effects effects are suppressed due to accidental numerical cancellations that occur at $13 \mathrm{TeV}$ and the typical $x$ and $Q^{2}$ regions probed at the LHC.

\section{What is wrong with the PDFs?}

There are a large number of opportunities for errors to slip into any given set of calculations: from computer code bugs to the addition of inconsistent data. The first place to check for problems is to check the codes used for calculation. ZTOP has been continuously checked for over 13 years, and reproduces old results exactly. One bug that was found early in this comparison was, like CTEQ 4, LHAPDF 5 [18] miscalculated $\alpha_{s}$ when run in multisets mode. ${ }^{1}$ This was corrected in LHAPDF 6. Another observation is that there are $<0.1 \%$ differences between PDFs returned from LHAPDF version of CTEQ 14, and the CTEQ interface to CTEQ 14. But be warned: in a NLO calculation, which has large numerical cancellations, it can take millions of Monte Carlo events to converge to the same result between the LHAPDF and CTEQ interfaces.

Another obvious possible solution to consider is that every PDF group has been sloppy in its LO fit. After all, the LO, NLO, and NNLO fits are independent, and there has not been a real emphasis on LO since 2002. This is something that needs to be examined by every group. It is difficult to trust the fitting procedures extended to higher orders if we cannot reproduce known results. However, it is logically possible that only NNLO (and maybe NLO) PDFs are valid, and for LO we must go back to using CTEQ 6L1.

Given that the disparities only arise in the newer PDF sets, the obvious questions to ask are what changed between 2002 and today, and why hasn't this been noticed before? The answer to the second question is that there has been a drought of LO PDFs for many years prior to the sets listed here. Hence, there was no opportunity to perform these checks with the newer fitting techniques. While calculations and fitting methods have evolved, the biggest change has been that the addition of jet data from the LHC, and final fits from HERA at DESY, have begun to heavily influence the fits of proton parton distribution functions.

Given the influx of new data sets in the fits I sought to determine what would happen if I removed the new data. NNPDF 3.0 includes sets that claim to fit the PDFs without LHC data (effectively no new jet data), or with HERA-only data. In the first part of Tab. 2 I recalculate at NLO using NNPDF 3.0 sets. The deviations do not change when LHC data is removed - which is curious, since jets data should change the NLO result; the gluon is best measured with jets data. A HERA-only fit by NNPDF seems even worse, which is strange given that HERA data is DIS-like. One caveat is that the NNPDF fit of HERA data is not consistent with the HERAPDF fit of HERA data shown in the second part of Tab. 2, so it should probably not be used for comparison. Unfortunately, there is no public LO version of the PDF fits with data sets removed. To truly make a comparison, the PDF groups need to provide the LO complement.

\footnotetext{
${ }^{1}$ Specifically, in LHAPDF 5 multiset mode, $\alpha_{s}$ was calculated for the first loaded PDF, and used for all other PDFs, even if the order should have been different.
} 
Table 2. Inclusive LO and NLO $t$-channel single-top-quark cross section $(t+\bar{t})$ calculated for run II of the Tevatron $(\sqrt{S}=1.96 \mathrm{TeV})$ with variations from NNPDF and HERAPDF. Note: There are no LO variation sets - making direct comparison of LO to NLO difficult.

\begin{tabular}{llll} 
PDF & LO $(\mathrm{pb})$ & NLO $(\mathrm{pb})$ & Notes \\
\hline NNPDF 3.0 lo/nlo & 2.33 & 2.21 & $5 \%$ deviation \\
NNPDF 3.0 (no LHC) & - & 2.22 & $5 \%$ deviation \\
NNPDF 3.0 (HERA) & - & 2.10 & $11 \%$ deviation \\
\hline HERAPDF 2.0 lo/nlo & 1.910 & 1.762 & $8 \%$ deviation \\
HERAPDF 2.0 (“JETS") & - & 1.830 & $4 \%-+c$, dijets, $\alpha_{s}$ data \\
\hline
\end{tabular}

Turning to the HERAPDF fits, where we would have expected DIS data to map onto DDIS data, the story is more subtle. While the baseline HERAPDF 2.0 fit has an $8 \%$ deviation between orders, it does not include all of the relevant data. HERAPDF also performed an alternate fit called "JETS" that includes the charm final state (the most closely related to single-top), and jets data which technically is differential DIS data. Both really should be included in the fit, and indeed the $\mathrm{LO} / \mathrm{NLO}$ difference is cut in half in this case. Still missing is a LO version of the "JETS" fit.

There is a lot of room for simple mistakes to be causing the large discrepancies between orders, with less room to explain why at most one NLO calculation is correct. However, we should also be asking: is something deeper is occurring?

Hidden behind the fitting methods for proton structure is an assumption that the degrees of freedom in the proton map to the universal PDF shape each group uses in a clear way. There is good reason to believe this is not the case for the gluons that appear in $t$-channel single-top-quark production. The reason is that $t$-channel single-top involves pure $V-A$ interactions at all vertices in both production and decay. This is what generates the famous angular correlations between final state particles that was used to aid in discovery $[1,2,8]$.

Current conservation means that only the left-handed polarization of the gluon is probed by $t$ channel single-top-quark. ${ }^{2}$ DIS charged-current data that goes into PDF fits is also left-handed, though neutral-current data has a right-handed contribution. Jets data completely mix left- and right-handed gluons. Single-top sees a polarized gluon in an unpolarized proton. Could the numerical discrepancies we are seeing be a consequence of ignoring spin-dependent effects in the mapping of data onto PDF fit functions? One numerical hint comes from another color-singlet exchange process described at ISMD XLVI [19]: Higgs production sees 2-5\% corrections from polarized gluons inside unpolarized protons. The large $\ln \left(m_{t}^{2} / m_{b}^{2}\right)$ terms which are losing their cancellations could easily boost this effect to $10-20 \%$, while in most other processes the effect would be hidden. This is not a proof, but it is suggestive that an effect which does have to be there could be large enough to see.

\section{Conclusions}

Are PDFs still consistent with Tevatron data? Of course they are. The $t$-channel single-top-quark data from the Tevatron is not sufficient to place strong bounds on the $b$ quark PDF. However, the calculations with modern PDFs that use significant amounts of LHC data as an input are much less in agreement than those that use older fits. Analytic constraints between LO and NLO PDFs are no longer satisfied, and NLO fits disagree with each other by up to $5 \sigma$. Something is clearly wrong, the question we now need to address is why is this failing?

\footnotetext{
${ }^{2}$ There is a negligible right-handed gluon contribution from the $b$ propagator spin-flip that goes like $\left(m_{b} / m_{t}\right)^{2}$.
} 
There are several avenues that should be followed to resolve the discrepancy discussed here. LO versions of fits with alternate data samples, such as removing LHC data or adding all HERA data, will help point toward which data sets might be driving the broken fits. Recalculation of the NNLO $t$-channel cross section using the DDIS scales will allow for validation of the NNLO fits. Will this be enough? It is possible the data is now so precise that we are seeing the breakdown of the way we map gluon degrees of freedom in the proton?

How might we use $t$-channel single-top-quark production as a foil for improving our PDFs in the future? One idea would be to use the analytic relationship between orders as a constraint on the fits. More speculatively, we may wish to consider fits are specialized to analytic constraints: e.g, fits of DIS data for DIS-like processes, fits of jets data for jets processes, etc. Ultimately, we should consider whether our data is now precise enough to consider alternate mappings of the gluon degrees of freedom in our fits that focus on these features. Perhaps we should be using spin-dependent PDFs even for unpolarized cross sections.

\section{Acknowledgments}

I would like to thank the ISMD organizers for an exceptional symposium and environment for lively discussions. This work was supported by the U.S. Department of Energy under award No. DESC0008347.

\section{References}

[1] T. Aaltonen et al. (CDF Collaboration), "First observation of electroweak single top-quark production,” Phys. Rev. Lett. 103, 092002 (2009) [arXiv:0903.0885 [hep-ex]].

[2] V. M. Abazov et al. (D0 Collaboration), "Observation of single top-quark production," Phys. Rev. Lett. 103, 092001 (2009) [arXiv:0903.0850 [hep-ex]].

[3] M. Brucherseifer, F. Caola, and K. Melnikov, "On the NNLO QCD corrections to single-top production at the LHC," Phys. Lett. B 736, 58 (2014) [arXiv:1404.7116 [hep-ph]].

[4] E. L. Berger, J. Gao, C.-P. Yuan, and H. X. Zhu, "NNLO QCD corrections to $t$-channel single top-quark production and decay," Phys. Rev. D 94, 071501 (2016) [arXiv:1606.08463 [hep-ph]].

[5] T. Stelzer, Z. Sullivan, and S. Willenbrock, "Single-top-quark production via $W$-gluon fusion at next-to-leading order," Phys. Rev. D 56, 5919 (1997) [hep-ph/9705398].

[6] Z. Sullivan, "Understanding single-top-quark production and jets at hadron colliders," Phys. Rev. D 70, 114012 (2004) [hep-ph/0408049].

[7] B. W. Harris, E. Laenen, L. Phaf, Z. Sullivan, and S. Weinzierl, "Fully differential singletop-quark cross section in next-to-leading order QCD," Phys. Rev. D 66, 054024 (2002) [hep$\mathrm{ph} / 0207055]$.

[8] Z. Sullivan, "Angular correlations in single-top-quark and $W j j$ production at next-to-leading order,” Phys. Rev. D 72, 094034 (2005) [hep-ph/0510224].

[9] A. Buckley, J. Ferrando, S. Lloyd, K. Nordström, B. Page, M. Rüfenacht, M. Schönherr, and G. Watt, "LHAPDF6: parton density access in the LHC precision era," Eur. Phys. J. C 75, 132 (2015) [arXiv:1412.7420 [hep-ph]].

[10] T. A. Aaltonen et al. (CDF and D0 Collaborations), "Tevatron Combination of Single-Top-Quark Cross Sections and Determination of the Magnitude of the Cabibbo-Kobayashi-Maskawa Matrix Element $\mathbf{V}_{\text {tb }}$, Phys. Rev. Lett. 115, 152003 (2015) [arXiv:1503.05027 [hep-ex]]. 
[11] H. L. Lai, J. Huston, S. Kuhlmann, F. I. Olness, J. F. Owens, D. E. Soper, W. K. Tung, and H. Weerts, "Improved parton distributions from global analysis of recent deep inelastic scattering and inclusive jet data," Phys. Rev. D 55, 1280 (1997) [hep-ph/9606399].

[12] H. L. Lai, J. Huston, S. Kuhlmann, J. Morfin, F. Olness, J. F. Owens, J. Pumplin, and W. K. Tung (CTEQ Collaboration), "Global QCD analysis of parton structure of the nucleon: CTEQ5 parton distributions,” Eur. Phys. J. C 12, 375 (2000) [hep-ph/9903282].

[13] J. Pumplin, D. R. Stump, J. Huston, H. L. Lai, P. M. Nadolsky, and W. K. Tung, "New generation of parton distributions with uncertainties from global QCD analysis," J. High Energy Phys. 07 (2002) 012 [hep-ph/0201195].

[14] J. M. Campbell, R. K. Ellis and F. Tramontano, "Single top production and decay at next-toleading order," Phys. Rev. D 70, 094012 (2004) [hep-ph/0408158].

[15] S. Dulat et al., "New parton distribution functions from a global analysis of quantum chromodynamics,” Phys. Rev. D 93, 033006 (2016) [arXiv:1506.07443 [hep-ph]].

[16] R. D. Ball et al. (NNPDF Collaboration), "Parton distributions for the LHC Run II," J. High Energy Phys. 04 (2015) 040 [arXiv:1410.8849 [hep-ph]].

[17] H. Abramowicz et al. (H1 and ZEUS Collaborations), "Combination of measurements of inclusive deep inelastic $e^{ \pm} p$ scattering cross sections and QCD analysis of HERA data," Eur. Phys. J. C 75, 580 (2015) [arXiv:1506.06042 [hep-ex]].

[18] M. R. Whalley, D. Bourilkov, and R. C. Group, "The Les Houches accord PDFs (LHAPDF) and LHAGLUE," hep-ph/0508110.

[19] D. Boer, "Multiple scattering aspects of gluon TMDs," presented at the XLVIth International Symposium on Multiparticle Dynamics, August 29-September 2, 2016, Jeju, South Korea. 